Este estudio analiza la neología léxica y la formación de palabras a partir de anglicismos en el lenguaje profesional del turismo para calibrar su dificultad para la traducción. Con este fin se ha conformado un corpus de informes de gestión turística y, a través de una extracción semiautomática, se ha realizado la identificación y clasificación de los candidatos a neologismos para observar los mecanismos de formación de palabras más utilizados y ofrecer una perspectiva de esas nuevas voces que sirvan de ayuda para la traducción de textos turísticos.

PALABRAS CLAVE: traducción, anglicismos, formación de palabras, turismo 2.0, neología léxica.

\title{
La neología del turismo 2.0: análisis de la creación léxica y retos para la traducción
}

Diana María González-Pastor

Universidad de València

Miguel Ángel Candel-Mora

Universitat Politècnica de València

\section{Neology in tourism 2.0: analysis of lexical} creation and its challenges for translation

This study analyses the lexical neology and the formation of words from anglicisms in the professional language of tourism to gauge their difficulty for translation. To this end, a corpus of tourist management reports has been compiled and through a semi-automatic extraction methodology, the identification and classification of the candidates for neologisms has been carried out to study the most frequently used word formation mechanisms and offer a perspective of these new words that helps the translation of tourism texts.

KEY WORDS: translation, anglicisms, word formation, tourism 2.0, lexical neology. 


\section{INTRODUCCIÓN}

Internet se ha convertido en la herramienta fundamental en la comunicación turística. La tecnología se ha revelado como un instrumento ideal que posibilita al turista la inmediatez, la accesibilidad en tiempo real de la información desde distintos dispositivos móviles y la participación activa en la experiencia turística y en su difusión. Por otro lado, para los profesionales del turismo, internet y las redes sociales ofrecen nuevas posibilidades para la mejora de la promoción y la mercadotecnia (Giménez Folqués, 2015). Del mismo modo, el análisis y la gestión cobran importancia a través de nuevos modelos en un sector en continua expansión y búsqueda de la diferenciación en la oferta y la creación de una experiencia única para el cliente.

La industria turística se enfrenta a grandes retos, puesto que nos hallamos en un tiempo de incertidumbre, intercambio y flujo dinámico de la información, donde la gobernanza de dicha información tiene un papel fundamental. Por estos motivos, entender el turismo es entender a la sociedad y entender a los turistas.

Según el barómetro Tripadvisor (20I6), el $89 \%$ de los viajeros de todo el mundo tiene en cuenta las opiniones en internet antes de realizar una reserva. La decisión está marcada por las opiniones digitales que pueda leer el turista (65\%), así como por las recomendaciones personales que se producen con el tradicional boca-oreja (48\%). En general, los turistas esperan recibir algún servicio añadido a su reserva, lo que puede venir de la mano de ofertas especiales. La tecnología es primordial también en este sentido, como pone de manifiesto el hecho de que el nuevo servicio más ofrecido en todo el mundo en los hoteles sea wifi gratis en la habitación. El 5 r\% de los viajeros a nivel global navega por internet durante sus viajes $y$ ha escrito una opinión durante o tras un viaje, ${ }^{\mathrm{I}}$ el $39 \%$ carga sus fotos en las redes sociales y el $33 \%$ encuentra actividades locales para su ocio a través del móvil (Tripadvisor, 20I6). ${ }^{2}$

La eclosión de las nuevas tecnologías aplicadas al turismo tiene una serie de consecuencias inmediatas para el turismo: los viajes son más rápidos, baratos y accesibles, lo que conlleva una serie de cambios en la cadena de valor y los conceptos de base de gestión y promoción de ventas. A corto plazo se producirá una digitalización de la vida social, por lo que el manejo de los datos personales constituye probablemente la mayor fuente de riqueza y poder en estos días para las empresas del sector3. La personalización de contenidos o la inteligencia artificial convertirán la «compra de turismo» en una experiencia intuitiva y sencilla. Se prevé que en 2024 ya no tendremos que estar comparando vuelos, hoteles y viajes, pues gracias a la inteligencia artificial contaremos con un agente electrónico que conocerá nuestras preferencias y nos acompañará a todas partes alojado en nuestro reloj.

Puesto que internet refleja completamente la organización real del sector turístico, es en este mismo canal donde se recoge toda la comunicación turística a través de las páginas web, ${ }^{3}$ que incluyen las nuevas formas de comunicación surgidas de internet. ${ }^{4}$ Como apunta

I La denominación «viajero social» empieza a usarse para describir a aquella persona que utiliza la tecnología (especialmente el teléfono móvil) antes, durante y después de su viaje para inspirarse, planificar y compartir sus experiencias y recuerdos.

2 Si bien el $72 \%$ de los viajeros en todo el mundo afirma que la posibilidad de efectuar reservas a través de un móvil es útil, solo el $25 \%$ de los alojamientos a nivel global está interactuando con sus clientes a través del móvil (Tripadvisor, 20I6).

3 De ahí que nuestro corpus sea íntegramente de fuentes en formato electrónico.

4 Blogs, posts, secciones de opiniones, etc., así como 
Calvi (2006: 53), «una página web es un medio que recoge materiales de todo tipo; un enorme contenedor en el que se va fraguando una amplia galaxia de subgéneros», por lo que nos encontramos con un lenguaje plagado de modalidades comunicativas y géneros diferentes, con objetivos y funciones distintas y que dan lugar a tipologías textuales muy variadas. ${ }^{5}$

En cuanto a la terminología y a los campos semánticos, las palabras del turismo se agrupan en los cuatro sectores clave: el alojamiento, el ocio o recreo, el viaje y la restauración. Estas cuatro actividades empresariales necesitan de una gestión eficaz y una promoción activa y competitiva. En consecuencia, esta investigación se aproxima al léxico del lenguaje turístico con un análisis desde el punto de vista de la formación de las palabras dentro del ámbito de la gestión profesional, cuyas características comunicativas exponemos en el siguiente apartado. Este trabajo profundiza en una investigación previa (González-Pastor y Candel-Mora, 20I7) con el objetivo de identificar las repercusiones que la neología léxica tiene para la traducción de textos turísticos.

\section{LA COMUNICACIÓN EN EL ÁMBITO DE LA GESTIÓN PROFESIONAL DEL TURISMO}

Dentro del sector turístico encontramos distintos niveles de especialización y modelos de comunicación. Algunos modelos recogen la complejidad del sector y distinguen tanto el ámbito comunicativo de los expertos como el

otros géneros de los que se derivan otros materiales previamente existentes en papel (documentos de viaje, catálogos, folletos...).

5 Véase la propuesta de clasificación de Calvi (2006) que recoge los géneros tradicionales del lenguaje turístico, formato en el que se presentan, función comunicativa, tipología textual y etapa de la experiencia turística a la que corresponde. de los turistas. Según Borrueco (2006), existen relaciones comunicativas simétricas, si se desarrollan entre profesionales o gestores del producto turístico; o asimétricas, ${ }^{6}$ en el caso de darse entre los gestores de producto y el turista o consumidor. La comunicación simétrica generada entre expertos suele presentar un alto grado de especialidad, mientras que la comunicación «entre especialistas y legos tiende a asimilarse más a discursos generales» (Gotti, 2006: 2I). Dicho esto, cabe señalar que también existen modalidades discursivas en las que la comunicación se efectúa entre expertos del sector turístico sobre temas de especialidad en los que el discurso resultante es informal y se acerca a los nuevos géneros digitales surgidos de internet (Carpi, 20I3).

Calvi (2008) recoge la tradicional división del estudio de las lenguas de especialidad que se articula en tres niveles fundamentales:

- La comunicación entre especialistas, caracterizada por la mayor densidad de conceptos y unidades terminológicas específicas;

- La comunicación dirigida a los semi-especialistas o especialistas en formación, en la que la finalidad didáctica determina una dilución de los contenidos específicos y el uso de procedimientos lingüísticos explicativos (reformulaciones, ejemplificaciones, etc.);

- La comunicación entre los especialistas y el público lego, es decir, la divulgación, que vehicula los contenidos especializados utilizando principalmente los recursos de la lengua común, y expresando los conceptos de la forma más sencilla posible (Calvi, 2008: I8I).

6 Gotti (2006) denomina estos dos planos comunicativos «comunicación interna» $\mathrm{y}$ «comunicación externa», entendiendo la primera como la comunicación que se produce entre especialistas de la misma profesión y externa como la comunicación entre especialistas. 
Suele ser en el ámbito profesional donde surgen nuevas ideas en la gestión, promoción y comercialización de productos turísticos que necesitan ser etiquetadas. Dichas etiquetas iniciales son susceptibles de desaparecer, evolucionar e incluso cristalizar en nuevas voces que traspasen el ámbito de la gestión profesional para formar parte de la lengua común. En cualquier caso, hemos de tener en cuenta que una voz resultará neológica «en función de la comunidad de habla que la utiliza o a la que va dirigida» (Sanmartín, 2009: 173).

El corpus objeto de nuestro análisis procede de textos electrónicos extraídos de los portales turísticos Hosteltur e Invattur de los años 20I4, 2015 y 20I6. Hosteltur 7 es un portal de noticias e información que está dirigido a los profesionales del sector turístico español con poder adquisitivo medio-alto o alto y elevada capacidad de decisión. Al utilizar los portales de internet para generar nuestro corpus estamos reflejando la comunicación que se establece de primera mano entre los profesionales del sector y posibilita «apostar por un texto con pluralidad, de voces y autores, con una articulación de la información distribuida por secciones» (Ibíd., I49). Su presencia en las redes sociales multiplica la difusión de sus contenidos que llegan a la totalidad del espectro turístico. ${ }^{8} \mathrm{El}$ mismo portal integra la Comunidad Hosteltur, ${ }^{9}$ el foro de discusión que está adaptado a todos los dispositivos móviles.

Por otro lado, Invattur, ${ }^{\mathrm{IO}}$ es un centro que sirve como plataforma de encuentro de todos

7 <http://www.hosteltur.com>.

8 Directivos de todos los hoteles y cadenas hoteleras de España, directivos de OTA, directores de agencias de viajes, aerolíneas, organismos oficiales y otros actores turísticos como las centrales de reservas o empresas de transporte.

$9<$ http://www.hosteltur.com/comunidad>.

ro Instituto Valenciano de Investigaciones Turísticas, $<$ http://invattur.gva.es>. los agentes del sector turístico y eje principal en la mejora del modelo turístico de la Comunidad Valenciana. Su portal en internet incluye un blog y un boletín periódico ${ }^{\mathrm{II}}$ que permiten acercar al sector turístico las novedades tecnológicas, transferir información sobre la evolución de la demanda y, en definitiva, contribuir a la aplicación de innovaciones que repercutan positivamente en la oferta turística valenciana. Finalmente, Segittur, ${ }^{\mathrm{I2}}$ es el portal de internet que recoge las acciones gubernamentales para estimular la innovación turística en España.

\section{LA NEOLOGÍA EN EL LENGUAJE DEL TURISMO}

La actividad turística se enmarca dentro de una gran variedad de sectores empresariales y engloba componentes temáticos muy variados y heterogéneos. La formación de palabras en turismo responde a dicha variedad y carácter multidisciplinar, por lo que su descripción y clasificación no resultan precisamente sencillas.

Desde los estudios de la lengua de especialidad del turismo en concreto, los neologismos aparecen definidos como «aquellas voces surgidas recientemente en una lengua, reconocidas mediante un criterio cronológico y lexicográfico (...), psicológico y de variación formal» (Estornell Pons, 2013: 40). ${ }^{13}$ Sin embargo, el estudio que presentamos a continuación se centra únicamente en uno de los procesos de creación de neologismos: los originados desde la lengua inglesa y puesto que el objetivo es estudiar su

II <http://invattur.gva.es/boletin-invat-tur >.

I2 <http://www.segittur.es/es/inicio/index.html>.

13 Aunque la exclusión del diccionario normativo es, por regla general, el criterio para el establecimiento del carácter neológico de un vocablo, este criterio es insuficiente (Estornell Pons, 2009), por lo que deben considerarse otros parámetros que contribuyan a determinar el carácter neológico de la palabra. 
tratamiento en traducción, se hace especial énfasis en los procesos de adaptación de esas nuevas voces, es decir, anglicismos adaptados, anglicismos no adaptados y calcos.

Estas unidades se crean en la lingua franca con el objetivo de facilitar el entendimiento y la comunicación entre los especialistas en muchos campos del saber (Guyot, 2010) y se incorporan al español y a otras lenguas sin ninguna adaptación. Este primer grupo de voces se caracteriza por un cierto grado de especialización. Suelen diferenciarse de los préstamos adaptados (Cabré, 2006: 234). El segundo grupo, el préstamo adaptado, es aquel que sufre una alteración morfológica, fónica u ortográfica para adecuarse a la lengua receptora (Castillo, 2002). Aunque es frecuente la distinción entre préstamos adoptados vs. préstamos adaptados, la adopción suele conllevar siempre un grado de adaptación, puesto que en el momento en que un préstamo se incorpora a la lengua se tiende a adaptarlo al sistema fónico y morfológico (Termcat, 2005). En cuanto al calco, este no solo se produce cuando se transfiere la palabra de una lengua a otra mediando la traducción de por medio, sino que, además, en el proceso se produce la sustitución de morfemas y el término puede acabar adquiriendo una nueva acepción. Según Gómez Capuz (2009: 7), «el calco es un tipo especial de préstamo que no imita la entidad fonética material del modelo extranjero sino otros aspectos más internos». La principal característica de los neologismos de forma reside en la formación de palabras a través de prefijación, sufijación, composición, lexicalización, conversión sintáctica, sintagmación, siglación, acronimia y abreviación. Los neologismos sintácticos, por su parte, implican un cambio de subcategoría gramatical en una base léxica. En el caso de los neologismos semánticos, lo que se modifica es el significado de la base léxica.
Según Alcaraz Varó et al. (2006), el turismo es un ámbito generador de palabras nuevas que se forman por el recurso de la composición y por la estrategia de conceder nuevas acepciones a palabras conocidas de la lengua común y que presentan cierta analogía. Así, por un lado, encontramos que son múltiples los compuestos que surgen para etiquetar nuevos deportes ${ }^{\mathrm{I}}$ relacionados con el turismo (hidrotrineo, heliexcursión ${ }^{15}$ ), nuevas formas de turismo (astroturismo, oleoturismo), nuevos productos turísticos (vivienda vacacional con servicios adicionales), así como formaciones que describen las nuevas formas de planificación, gestión y organización turística (empresas de tecnología hotelera, ${ }^{16}$ red de coche compartido, ${ }^{17}$ búsqueda multidestino).

Por otro lado, la lengua inglesa juega un papel fundamental como lingua franca en los textos turísticos. Es frecuente encontrar extranjerismos, especialmente anglicismos (Lorenzo, I996) provenientes de voces inglesas que ya forman parte de nuestro lenguaje cotidiano (overbooking, ferri). El hecho de que el inglés se haya impuesto como lengua global hace que los nuevos giros, palabras y términos que se van acuñando en el sector turístico adopten la denominación inglesa. Los préstamos pueden ser no adaptados, es decir, transferidos íntegramente en inglés a la lengua meta o adaptados, de modo parcial, cuando solo un lexema se adapta a la lengua meta (punto selfie). En otros casos, la

I4 Véase el estudio de Estornell Pons (2013) sobre la neología léxica del turismo activo.

I5 Heliexcursión es una palabra creada por acronimia (se unen la palabra truncada helicóptero y un lexema completo).

I6 Se trata de empresas de sistemas centralizados de reservas, software para gestión de ingresos y marketing digital.

${ }_{7}$ La empresa Blablacar lidera este nuevo tipo de consumo colaborativo. 
influencia de la lengua inglesa queda patente a través de calcos (turoperador) u otro tipo de formaciones híbridas o construcciones formadas a partir de la unión de un lexema y una abreviatura (tourismmkt). Este estudio presta especial atención a los anglicismos y a aquellas unidades léxicas que siguen percibiéndose como palabras extranjeras a pesar de aparecer junto a palabras parcialmente adaptadas. Como indica Berná Sicilia (201r: 286), esto se debe a que «las nuevas voces han reproducido los procesos internos de creación léxica en español».

Otros elementos neológicos de gran importancia y alta frecuencia en el lenguaje turístico son las siglas y los acrónimos, referidos a nuevas organizaciones y entidades turísticas (EEOO, Escuela Europea de Cata de Aceite y Oleocultura, OTA, online travel agency) o ferias del sector (ITMM, International Travel Media Meeting).

Todas estas formaciones neológicas pueden tener un carácter temporal y variar en su morfología y su uso, por lo que su estabilidad y repetición resultan fundamentales para determinar si nos encontramos ante elecciones estilísticas personales y estrategias discursivas del especialista o usos generalizados por parte de los expertos en turismo. Sin embargo, no es nuestro objetivo establecer el carácter neológico de los términos que a continuación analizamos, sino dibujar el panorama de las nuevas voces potencialmente neológicas y que no están codificadas en los diccionarios.

\section{ANÁLISIS Y DISCUSIÓN}

Para la identificación, extracción y posterior análisis de anglicismos en el lenguaje de la gestión profesional del turismo se procedió a la elaboración de un corpus compuesto por informes sobre gestión turística elaborados por empresas del sector o empresas de consultoría turística independientes, así como publicaciones de noticias y notas de prensa emitidas por estas mismas instituciones, de modo que el lenguaje objeto de estudio estuviera lo más actualizado posible. Los textos proceden de las plataformas Hosteltur, Invattur y Segittur, que están destinadas a la difusión del conocimiento en la gestión profesional del turismo. Posteriormente se diseñó una estrategia de extracción computacional semiautomática de candidatos a neologismo en el corpus formado por 84 textos, $y$ un total de $730.55^{2}$ palabras. Aunque existen diferentes formas de extraer neologismos desde un corpus de textos, todo parece indicar que se pueden concentrar en tres enfoques distintos (Janssen, 2009): mediante el uso de listas de exclusión con palabras conocidas; mediante el uso de patrones lingüísticos y construcciones donde aparecen habitualmente los neologismos; y mediante el análisis de listados de frecuencias y ocurrencias del corpus. A este análisis se añade las diversas variantes y la combinación de metodologías entre los tres métodos de detección.

Tabla 1. Estadísticas del corpus

\begin{tabular}{|c|c|}
\hline tokens (running words) in text & $73055^{2}$ \\
\hline tokens used for word list & 663399 \\
\hline types (distinct words) & 28733 \\
\hline type/token ratio (TTR) & $4,33 \mathrm{II} 79 \mathrm{I} 42$ \\
\hline
\end{tabular}

En cualquier caso, a pesar del número de herramientas, los avances en tecnología y lingüística computacional y los diversos enfoques a la extracción de neologismos, se concluye que sigue siendo necesaria la intervención humana en la detección de candidatos a neologismos (Janssen, 2009). Por ello, este trabajo ha utilizado una metodología híbrida consistente en 
la combinación de resultados obtenidos por un análisis del corpus con Wordsmith Tools y la observación de las listas de frecuencias de palabras con la extracción automática por medio de la herramienta Neotag, ${ }^{18}$ a la que se ha añadido la revisión humana por expertos lingüistas para confirmar la existencia del anglicismo y proceder al consiguiente análisis y discusión sobre el método de formación seguido. Para la revisión humana y detección de los candidatos, hemos tomado como referencia los criterios de Cabré (I992), que propone, además del criterio lexicográfico, el criterio diacrónico (la aparición reciente del término), el criterio de inestabilidad formal (variación morfológica, gráfica o fonética) y semántica y el criterio psicológico, por el que una unidad se puede considerar neológica si los hablantes la perciben como nueva.

Cabe destacar que, al centrar el trabajo en la extracción de anglicismos, el procesado de los textos con el etiquetador de Neotag simplificó considerablemente las tareas de extracción de candidatos a neologismos puesto que una de las etiquetas que ofrece el programa es type:foreign utilizada para etiquetar los elementos no identificados inicialmente como una categoría gramatical reconocible por el sistema Neotag.

Del corpus de textos publicados por los profesionales del turismo se extrajeron inicialmente un total de 378 candidatos. La mayoría de los candidatos que arroja la extracción de neologismos son préstamos no adaptados ( $77 \%$ ), mientras que la presencia de formas adaptadas y calcos es muy inferior.

Asimismo, una vez extraídos los candidatos, se procedió a clasificarlos por áreas temáticas para proceder posteriormente a su estudio desde el punto de vista de su creación léxica. La

I8 Disponible en <http://marke.upf.edu/ neotag/?action=documentation $>$. propuesta de clasificación temática con las áreas identificadas es la siguiente:

- Formas de hacer turismo

- Perfiles de turista

- Marketing, promoción y venta por internet

- Gestión hotelera

- Innovación tecnológica

La categoría que presenta más de la mitad de los candidatos a neologismos es la de innovación tecnológica, seguida de la de marketing, promoción y venta por internet, como se observa en la figura r:

\section{Distribución de neologismos por area temática}

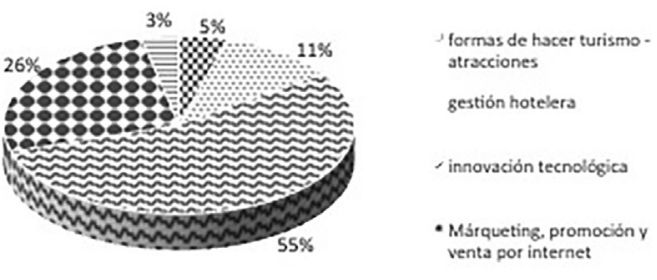

Figura I. Distribución de neologismos por área temática

Sin embargo, las áreas relacionadas con los nuevos modelos de negocio y gestión hotelera, los nuevos perfiles de turista y las nuevas atracciones turísticas presentan cantidades muy inferiores de neologismos, lo que muestra la relevancia que tienen actualmente internet, las nuevas tecnologías y la promoción y venta en la gestión profesional a través de este canal en este sector.

En general, los anglicismos adaptados y los calcos son los que registran un menor número en nuestro corpus por lo que respecta al mecanismo de creación léxica, lo cual confirma la dificultad de adaptación morfológica o fonética de vocablos extranjeros. Así, nos hemos encontrado con grupos de palabras en los que 
Tabla 2. Número de candidatos por áreas temáticas y su mecanismo de creación léxica.

\begin{tabular}{lllll}
\hline Área temática & Calco & No adaptado & Adaptado & Total \\
\hline formas de hacer turismo -atracciones & 4 & 9 & 4 & I7 \\
\hline gestión hotelera & I & 3 I & 9 & $4 \mathrm{I}$ \\
\hline innovación tecnológica & 32 & I54 & 2 I & 207 \\
\hline Marketing, promoción y venta por Internet & 6 & 87 & 7 & IO \\
\hline perfiles de turista & I & 9 & 3 & I3 \\
\hline Total & 44 & 290 & 44 & 37 \\
\hline
\end{tabular}

existe gran inestabilidad en cuanto a su forma, que da lugar a variación morfológica, gráfica, y fonética. Si observamos cuál es el mecanismo de creación léxica en relación con su clasificación temática (ver tabla 2) podemos comprobar que en todos los campos semánticos identificados se mantiene la tendencia del uso del anglicismo no adaptado muy por delante de las formas adaptadas y los calcos.

A continuación, realizamos un análisis exhaustivo de los neologismos contextualizándolos en las categorías temáticas propuestas. Este análisis incluye información acerca de los mecanismos de creación más relevantes y proporciona ejemplos ilustrativos de las nuevas palabras del turismo y de las traducciones documentadas.

\section{CLASIFICACIÓN DE LOS NEOLOGISMOS DEL LENGUAJE TURÍSTICO}

\section{Formas de hacer turismo}

Por lo que respecta a los distintos productos turísticos y su gestión se han registrado I7 neologismos, 9 de ellos no adaptados, 4 adaptados y 4 calcos.

En esta categoría destacan dos extranjeris- mos relacionados: city break ${ }^{19}$ y smart city. ${ }^{20} \mathrm{El}$ primero es una unidad neológica procedente del término turístico break, ${ }^{2 \mathrm{I}}$ que alude a un «viaje de corta duración, (normalmente de unos días o de un fin de semana) y constituye un paréntesis en pleno trabajo» (Alcaraz Varó et al., 2006: 68). Cuando este paréntesis está enfocado al turismo urbano en una ciudad del propio país como de algún país vecino, realizamos un city break. ${ }^{22} \mathrm{En}$ español disponemos del vocablo «escapada» que el DRAE define como «abandono temporal de las ocupaciones habituales, generalmente con objeto de divertirse o distraerse». No obstante, la voz «escapada» no es del todo equiparable semánticamente a city break, pues es un término general que suele combinarse con la duración del viaje (escapada de fin de semana), ${ }^{23}$ o el periodo en el que se viaja (escapada de Nochevieja y Año

i9 <http://www.hosteltur.com/I42020_turistas-alemanes-piden-turismo-sostenible-city-breaks.html>.

20 <http://www.hosteltur.com/I52I70_turismo-urbano-como-gestionar-marca-ciudad.html $>$.

${ }_{21}$ En español se usa el término escapada.

${ }^{22}$ Este producto ha experimentado una rápida expansión debido a un cambio de comportamiento en el turista que prefiere viajes más cortos, y también al aumento de rutas aéreas y tarifas más económicas.

${ }_{23}$ <http://www.hosteltur.com/I8980o_portugal-atenas-budapest-escapadas-economicas-temporada-20I3-I4. html>. 
Nuevo ${ }^{24}$ y no necesariamente implica un destino urbano. Sin embargo, los expertos también utilizan «escapada» en este sentido:

La aplicación móvil HotelTonight ha elaborado un informe sobre el comportamiento del viajero que utiliza el móvil a la hora de organizar sus escapadas, y sobre el estado actual del mercado de hoteles de última hora. (...) El estudio elaborado por HotelTonight revela que en otoño los destinos europeos más visitados serán Londres, París, Roma, Barcelona y Dublín; aunque los mejores para conseguir descuentos del 30-50\% en el alojamiento serán Edimburgo, Munich, Madrid, Sevilla y París ${ }^{25}$ (Hosteltur, 20I4).

Los expertos utilizan también las voces smart city ( Telefónica convertirá Valencia en una Smart City $\left.{ }^{26}\right)$ y ciudad inteligente indistintamente («Telefónica ha diseñado para Valencia la primera solución tecnológica de gestión integral que transformará la ciudad en una ciudad inteligente y totalmente conectada»). Estas voces, junto con sus derivados smart destination ${ }^{27} \mathrm{y}$ destino turístico inteligente, ${ }^{28}$ cohabitan en el lenguaje especializado del turismo y aluden a un destino innovador, consolidado sobre una infraestructura tecnológica de vanguardia, que garantice el desarrollo sostenible del territorio turístico, accesible para todos, que facilite la inte-

24 <http://www.hosteltur.com/I8589o_ranking-desti-

nos-buscados-nochevieja-ano-nuevo.html .

$25<$ http-viajero-europeo-reserva-hotel-ultima-hora. html>.

26 <http://invattur.gva.es/noticia/ telefonica-convertira-valencia-en-una-smart-city/>.

27 <http://www.segittur.es/es/sala-de-prensa/detalledocumento/E1-ministro-de-Industria-destaca-que-Espaalidera-a-nivel-mundial-la-conversin-de-destinos-tursticosen-Smart-Destinations-/\#.VNJdQWiG_X $4>$.

28 <http://invattur.gva.es/noticia/la-generalitat-estaintegrando-el-modelo-destino-turistico-inteligente-en-suestrategia/>. racción del visitante con el entorno e incremente la calidad de su experiencia en el destino. ${ }^{29}$

Otros candidatos a neologismo reseñables en esta área temática son: turismo de borrachera, voluntourism, astroturismo, red de coche compartido, bioturismo, experiencia premium, miradores de vértigo, gentrificación turística y free tours.

\section{Perfiles de turista}

La comunicación turística profesional en internet también contempla la aparición de nuevas formas de hacer turismo y nuevos perfiles de turista. La mayoría de estas formas neológicas son préstamos no adaptados.

Así, en esta categoría hemos registrado las formas no adaptadas mountainlikers ${ }^{30}$ y Millenials..$^{3 \mathrm{I}} \mathrm{El}$ primero hace referencia a los turistas amantes de los deportes de nieve y de montaña. $\mathrm{El}$ segundo término tiene su origen en el término millenium y se refiere a un grupo determinado de turistas que pertenecen la generación que se hizo mayor de edad con la entrada del nuevo milenio. Esta generación del milenio es experta en redes sociales y en compartir experiencias positivas o negativas, además de ser muy susceptibles a las políticas de premios y recompensas que ofrecen las empresas hoteleras.

En el lenguaje turístico profesional son abundantes las formaciones surgidas a través del calco de la palabra o grupo de palabras que no solo deben su significado a la lengua extranjera, sino también su forma, que es una traducción de estructura del sintagma original, como ocurre con la voz prosumidor. Según Wikipedia, la voz prosumidor, también conocida como prosu-

29 Definición propuesta por Segittur.

$3^{\circ}<$ http://www.hosteltur.com/comunidad/ooziIo_ montanas-y-turistas-los-nuevos-mountainlikers.html> .

$3^{\mathrm{I}} \quad<\mathrm{http}: / /$ www.hosteltur.com/I6696o_fidelizacionhotelera-millennials-experiencia-es-lo-cuenta.html>. 
Tabla 3. Mecanismos de creación léxica en el área temática del marketing, promoción y venta por internet.

\begin{tabular}{lllll} 
Neologismos & Mecanismos & & \\
\hline Area temática & Calco & No adaptado & Adaptado & Total \\
\hline Marketing, promoción y venta por internet & 6 & 87 & 7 & Ioo \\
\hline
\end{tabular}

mer, es un acrónimo formado por la fusión de las palabras en inglés productor (productor) y consumer (consumidor). Se trata de un término utilizado en ámbitos muy diferentes, que también hemos registrado en el ámbito del lenguaje turístico con la siguiente acepción:

El prosumidor o prosumer (...), en lo referido a internet y los viajes, se trata de un individuo que puede dedicar horas y horas, jornadas y jornadas, a buscar en múltiples buscadores, metabuscadores y webs ese chollo de viaje del que podrá presumir durante semanas ante amigos, familiares y vecinos (Hosteltur, 20I4). $3^{2}$

Otros vocablos registrados en esta categoría y que tienen especial interés son: turista digital, viajero Pack, Dinkies (proviene de Double income no kids y hace referencia a las parejas con doble renta y sin hijos), Panks (profesional aunts no kids), y foodies (turistas gastronómicos).

\section{Marketing, promoción y venta por internet}

La mayoría de las unidades neológicas registradas en esta categoría semántica son préstamos que proceden de la lengua inglesa. Estas unidades se crean en lengua inglesa con el objetivo de facilitar el entendimiento y la comunicación entre los especialistas y se filtran al español (y a otras lenguas) sin ninguna adaptación (ver tabla 3).

\footnotetext{
$3^{2}$ <http://www.hosteltur.com/I30620_internet-hapuesto-patas-arriba-al-sector-agencias-viajes.html>.
}

Este grupo de voces se caracteriza por un cierto grado de especialización. Es el caso de voces como sharing economy. Observamos el uso del préstamo puro para dar cuenta de la actividad de las empresas que utilizan las nuevas tecnologías para of recer nuevos modelos de negocio ${ }^{33}$ que dan respuesta a las necesidades de los nuevos perfiles de turista. Aunque en español existen las voces «economía colaborativa» $\mathrm{y}$ «consumo colaborativo», entre los expertos se alterna frecuentemente el uso del extranjerismo sharing economy con la voz en español. ${ }^{34}$

En este mismo ámbito del marketing también se ha documentado la unidad cross-selling, que es la táctica que persigue vender productos complementarios a los que un cliente consume o pretende consumir. Aunque en español el Diccionario de términos económicos, financieros y comerciales de Alcaraz Varó y Hughes (r999) recoge el término cross-sell, cuyo equivalente normativo en español es «venta cruzada», el especialista en turismo prefiere utilizar la denominación inglesa, por lo que ambas voces en inglés y español se alternan entre los especialistas del marketing turístico. Del mismo modo, la forma inglesa revenue management es la preferida por los especialistas del sector hotelero:

\section{(...), empresa especializada en servicios de}

33 Un caso representativo es la empresa Airbnb, portal líder de alojamiento colaborativo.

34 También se ha registrado el uso del término turismo colaborativo, que se alterna con la voz turismo $22 p$. Véase <http://www.hosteltur.com/r6456o_nh-obradoiro-entracartera-premium-cadena.html>. 
revenue management y distribución online para hoteles, ha reforzado su estructura comercial y de expansión con varias incorporaciones. Así ha nombrado a (...) como director comercial. Asimismo, también ha nombrado como director de revenue management a (...), quien estará al frente de un equipo integrado por más de 20 revenue managers, con el objetivo de potenciar la distribución y resultados de los hoteles clientes. 35

Este término y sus derivados hacen referencia a la aplicación de herramientas de análisis que predicen el comportamiento del consumidor. Aplicado al sector hotelero, se refiere a las diferentes tácticas y estrategias para vender la habitación correcta, al cliente correcto, en el momento correcto, al precio correcto y a través del canal correcto. El hecho de que sea una técnica relativamente nueva en el ámbito de la hotelería, y aún más en España y otros países hispanohablantes, ha favorecido la adopción de los términos en inglés. No obstante, se ha documentado el neologismo «gestión de ingresos», ${ }^{36}$ término que resulta de una traducción literal de la expresión en lengua inglesa, también en otros campos del turismo como el de las aerolíneas. ${ }^{37}$

Las redes sociales y su creciente desarrollo en el sector turístico y la industria hotelera quedan patentes con el uso de las nuevas voces social commerce $^{38}$ y social sharing point. Se trata de dos extranjerismos no adaptados provenientes del inglés y creados por composición con el

35 <http://www.hosteltur.com/hemeroteca\#revenue+ management>.

36 <http://comunidad.hosteltur.com/post/20I2oI-I6-revenue-management-como-modelo-de-gestinintegral-de-ventas.html>.

$37<$ http://www.hosteltur.com/ hemeroteca\#gestion+ingresos>.

${ }^{8}$ Este término fue acuñado por la empresa de tecnología Yahoo! en 2005 para describir un conjunto de herramientas de compra colaborativa (listas compartidas, valoraciones de usuarios, y otro tipo de consejos de los usuarios). adjetivo «social», del que han surgido incontables voces relativas a la comunicación e interacción de las personas a través de la tecnología y las comunicaciones móviles. ${ }^{39}$ Estamos, pues, ante una palabra clave, muy recurrente y de gran productividad. El DRAE todavía no recoge la nueva acepción de este adjetivo, y lo define, en su sentido más amplio, como «perteneciente o relativo a la sociedad».

La enciclopedia electrónica Wikipedia recoge «comercio social» como «una ramificación del comercio electrónico que supone el uso de redes sociales para ayudar en la compra y venta en línea de productos y servicios»..$^{\circ}$ En la industria turística, las redes sociales se han consolidado no solo como un canal de comunicación y promoción de las empresas. A su vez, son el foro de comunicación de los turistas, donde se comparten impresiones sobre determinados establecimientos o productos turísticos y se intercambian opiniones que resultan claves a la hora de realizar una reserva.

En este mismo sentido se utiliza social sharing point t $_{\text {unidad formada por composición }}$ que describe un lugar especialmente designado en un establecimiento turístico, generalmente un hotel para que el viajero realice sus fotografías y las comparta en la red. Actualmente, los hoteles están estimulando a sus clientes para que sean los protagonistas de este tipo de publicidad en las redes sociales ofreciéndoles a cambio puntos en sus programas de fidelización $o$ algún otro tipo de compensación. ${ }^{4 \mathrm{I}}$

39 Social media, social networks, entre otras. Véase <http://mglobalmarketing.es/ blog/IoI-anglicismos-mas-utilizados-en-marketing>.

$4^{\circ}<$ https://es.wikipedia.org/wiki/Comercio_social>.

4r La cadena de hoteles Barceló, por ejemplo, realiza concursos a través de su página de Facebook animando a los turistas a hacerse fotos en algún rincón de sus hoteles y realizando comentarios. A cambio el viajero entra en el sorteo de un circuito spa en el hotel. 
Otro anglicismo que hemos registrado en nuestro estudio es blogtrip..$^{42}$ Se trata de una herramienta novedosa y muy interesante para la comunicación y la promoción turística en internet. El término blogtrip (también registrado como blog trip) se utiliza desde hace años para designar un tipo de viaje en el que las oficinas de turismo o empresas de un determinado sector invitan a una serie de blogueros ${ }^{43}$ influyentes a disfrutar de un destino a cambio de que estos publiquen después sus impresiones, su valoración y toda la información relacionada con la experiencia en sus respectivos blogs. Asimismo, las nuevas formas de comunicación y promoción en el sector turístico en internet han dado lugar a una nueva profesión: los videotellers, ${ }^{44}$ especialistas en creación de videos y contenidos que las empresas comparten en la red y que pueden acabar convirtiéndose en virales. Se trata de una tendencia global de las empresas turísticas, que hacen uso de los videos promocionales a modo de catálogo de viajes.

En los casos que se documentan a continuación encontramos una adaptación parcial en la que únicamente el primer lexema de la formación se ha adaptado al español. Así, por ejemplo, notificación push es una forma anglicada que proviene de la voz inglesa push notification. Notification y «notificación» son fónica, morfológica y ortográficamente casi idénticas, por lo que la unidad léxica reúne las condiciones para ser adaptada en la lengua receptora. La Wikipedia recoge el término Tecnología Push ${ }^{45}$ (en contraposición a Tecnología Pull) y utiliza el

$42 \quad<$ http://www.hosteltur.com/I3315o_blogtrips-vspresstrips-formulas-compatibles-excluyentes.html>.

43 Blog y bloguero/a sí están normalizados en la última edición del DRAE.

$44<\mathrm{http} / / /$ www.hosteltur.com/I3I2IO_videotellersnuevos-prescriptores-viajes.html>.

$45<$ http://es.wikipedia.org/wiki/

Tecnolog\% $\mathrm{C}_{3} \% A D a \_$Push $>$.

término mensaje para referirse a la información transmitida, no así el término notificación:

La mecánica de Flashbooking ${ }^{46}$ es simple: una vez que el viajero descarga la aplicación en su móvil, debe darse de alta, proporcionando información como lugar y fecha del viaje tipo de cliente (viaja en familia, solo, con amigos, etc.), preferencias de ocio (turismo náutico, de aventuras, gastronómico, cultural, etc.), y precio máximo que desea pagar por actividad. Con estos datos se crea un perfil único que segmenta los resultados de búsqueda y genera notificaciones push: una vez seleccionada la actividad de ocio deseada, el turista puede elegir entre tres opciones: Comprar Ahora, Fijar un Descuento o Seguir (Invattur, 20I4).47

Por último, un calco que resulta especialmente interesante es el de «influenciador de viajes», (trip influencer), que es aquella persona u organización que constituye un líder de opinión en internet en cuanto a destinos turísticos:

Importantes bloggers e influenciadores en viajes y turismo de talla nacional e internacional se dieron cita ayer en el Social Media \& DMo's, celebrado en el Auditorio del Mar Rojo del Oceanográfico de Valencia. El evento, organizado por la Conselleria de Turismo, Cultura y Deporte y el Invattur, supuso una oportunidad única para conocer, de la mano de profesionales y marcas de primer nivel, las posibilidades del social media y el papel de los influenciadores en la promoción de destinos (Invattur, 2015)..$^{8}$

Otros neologismos dignos de mención en

46 Flashbooking es una aplicación turística de compras sociales, que permite a los turistas compartir sus planes de ocio y ofertas en destino, promoviendo las compras en grupo para conseguir mejores precios y descuentos.

47 <http://www.invattur.org/noticias/flashbooking-laprimera-app-de-compra-social-en-turismo >.

$4^{8}$ <http://www.invattur.org/noticias/influenciadoresde-primer-nivel-se-renen-en-el-social-media-dmos $>$. 
esta categoría son: webs de comentarios, reputación online y publicidad nativa.

\section{Gestión hotelera}

En nuestro estudio también se documentan varias unidades que pueden catalogarse dentro del ámbito de la gestión profesional hotelera, como por ejemplo punto selfie y hoteles adults only. En el primer caso, la nueva voz alude a un lugar o zona de un hotel en la que los turistas que allí se alojan pueden realizarse un autorretrato hecho con un teléfono de última generación:

Con el objetivo de reunir esta publicidad gratuita de los clientes y mejorar su estancia, algunos hoteles aconsejan ahora dónde hacerse los mejores selfies y convocan concursos animándoles a compartirlos en las redes sociales para así ganar una estancia gratis. El hotel Grand Bretagne en Atenas, por su parte, tiene delimitado un 'punto selfie' en su azotea, estratégicamente situado para que los clientes puedan sacarse la mejor foto con la Acrópolis a sus espaldas (Hosteltur, 20I4).49

De nuevo, nos encontramos con el lexema «punto» como equivalente a «lugar» o «sitio». Para la Fundéu la evidencia del uso abrumador de la voz inglesa selfie, tanto en medios hablados como escritos, sugiere la pertinencia de proponer la adaptación selfi (plural selfis), que refleja en español la pronunciación de este término inglés y no ofrece problemas de adaptación a nuestro sistema..$^{\circ}$ Sin embargo, en el ámbito profesional del turismo observamos una predilección y uso recurrente de la forma inglesa sel-

49 <http://www.hosteltur.com/I7056o_hoteles-convierten-selfies-experiencia-vacacional-promocion.html>.

50 También existe el neologismo palo selfi que hace referencia al soporte que se usa para realizarse autofotos. Esta última palabra es una alternativa adecuada en español para selfie en inglés. fie..$^{5 \mathrm{E}} \mathrm{El}$ segundo caso de unidad potencialmente neológica es la expresión hoteles adults only, ${ }^{52}$ establecimientos que ofrecen una selección de productos diferentes a los del hotel convencional y aportan un valor muy distinto al segmento de parejas, grupos, familias y lunas de miel. A diferencia de lo que observamos con punto self$i e_{\text {s }}$ en este caso existe variación denominativa y los profesionales del turismo alternan el préstamo adaptado parcialmente con las expresiones hotel only adults y el sintagma en español hoteles para adultos.

Del mismo modo que ocurre con social, hemos registrado el uso frecuente del adjetivo inglés premium para formar compuestos sintagmáticos con estructura compuesta por nombre más adjetivo que aparecen en el lenguaje profesional del turismo, tales como hotel premium, experiencia premium, ${ }^{33}$ cartera premium ${ }^{54} \mathrm{O}$ servicio premium. ${ }^{55} \mathrm{El}$ Diccionario de Neologismos online del Observatori de Neologia define premium como un producto o servicio «que es de la calidad más alta de una categoría». En todas estas designaciones, ambos elementos no cobran ningún sentido más allá del que se les atribuye individualmente en el diccionario. Este préstamo puede plantear dificultades en su uso por lo extraño de su pronunciación en castellano. La Fundéu ${ }^{56}$ recomienda evitar el anglicismo premium y sustituirlo por sus expre-

${ }^{5} \quad$ Elegida palabra del año 2013 por los diccionarios Oxford University.

$5^{2} \quad<$ http://www.hosteltur.com/16855o_hoteles-adultsonly-mejorar-destino.html>.

$53<\mathrm{http} / /$ www.hosteltur.com/I7212O_coche-electricodestinos-nueva-experiencia-premium.html>.

$54<\mathrm{http} / /$ www.hosteltur.com/I6456o_nh-obradoiroentra-cartera-premium-cadena.html>.

55 <http://www.segittur.es/opencms/export/sites/ segitur/.content/galerias/descargas/Informe-Sistema-deMensajera-Instantnea-para-la-empresa-ok.pdf $>$.

$5^{6}<\mathrm{http} / /$ www.fundeu.es/recomendacion/ premium-anglicismo-innecesario-768>.

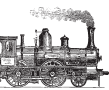


siones equivalentes en español según el nombre que califiquen: productos, marcas, instalaciones o servicios «de lujo o de gama alta».

94
Otros anglicismos y creaciones léxicas que destacan en este área temática son: room service, hostel, hoteles cápsula, habitación cápsula, vivienda vacacional con servicios adicionales, empresas de tecnología hotelera, pisos turísticos, resort subacuático. Asimismo, debemos mencionar el término ryanaización, que es un calco procedente del nombre de la aerolínea Ryanair. Este neologismo muestra las infinitas posibilidades de creación del lenguaje de los profesionales, que expresa la idea de modelo de negocio de bajo coste añadiendo un sufijo nominalizador en español a partir del anglicismo.

\section{Innovación tecnológica}

Esta categoría es la que contiene mayor número de neologismos con 207 casos, principalmente préstamos no adaptados. La mayoría de estos designan herramientas tecnológicas o sistemas en internet habituales en la gestión profesional, o de uso por parte del turista (apps, cloud, offline, bluetooth, tablet, PDA, microblogs, smartglasses).

Hemos observado que algunos neologismos de nuestro corpus presentan inestabilidad formal, como el ejemplo de cluster, que presenta otra forma adaptada (clúster), si bien la forma no adaptada sin acento predomina sobre la adaptada. Hay documentadas más de una decena de traducciones para la palabra cluster, según el ámbito especializado en el que se use (agrupación, agrupamiento, conglomerado, entramado, glomérulo, haz, etc.) pero en español se usa cluster o clúster (González, 2012: 269).

Los calcos registrados en nuestro análisis pertenecen, en su mayoría, al ámbito de la tecnología. Destaca el vocablo smartphone, que también experimenta una gran inestabilidad morfológica. El uso de la forma plural y singular se alternan para reproducir el vocablo inglés. Los especialistas del turismo utilizan habitualmente la palabra smartphone aunque no la escriben correctamente, pues se han registrado otras unidades léxicas como smarpthone, smarthphone, smarthpone, entre otras. Un ejemplo de calco constituye la palabra warables, que hace referencia a la tecnología «vestible o ponible» y que procede el inglés wearable.

En general encontramos un grupo de verbos que hace referencia al uso de las redes sociales en el turismo que han sido adaptados desde la forma inglesa, como son instagramear, loguearse, retuitear, trackear, chatear, hackear.

Entre los procesos más productivos cabe destacar los que contienen como raíz blog y web. Estos lexemas generan tanto nuevos anglicismos como calcos y formas adaptadas, lo que se traduce en una multiplicidad de designaciones adaptadas y no adaptadas para un mismo referente (blog, blogger, bloguer, bloguero, blogspot, blogosfera y webapp, webcam, webdesign, weblog, webmobile, webpark...). Sin embargo, la raíz web no genera derivados adaptados parcial o totalmente al español, sino que únicamente se observa el lexema con sus derivados, también anglicismos.

\section{CONCLUSIONES}

Las voces recogidas en el presente estudio pertenecen a las nuevas formas de entender, gestionar y hacer turismo. En muchos casos, estamos ante tendencias de la industria turística que provocan la necesidad de nombrar realidades nuevas. Estas tendencias son un reflejo del mundo en el que vivimos, favorable a la creación de estos términos que corresponden a nuevos conceptos, tanto materiales como intelectuales 
(Guerrero Ramos, 1995/2010). En este estudio hemos constatado un grupo mayoritario de nuevas voces vinculadas al uso de internet, las redes sociales y las tecnologías móviles en la actividad turística.

internet y el auge de las tecnologías digitales han revolucionado el modo en que viajamos y hacemos turismo: desde cómo planeamos a cómo reservamos, pasando por cómo disfrutamos de las vacaciones y cómo compartimos nuestra experiencia con nuestra familia y amigos. Las experiencias que vivimos ya no solo se quedan en nuestro círculo de amigos, sino que las compartimos a través de los nuevos medios para facilitar la elección a otros viajeros. Numerosas aplicaciones nos ayudan a comparar precios y comprar nuestras vacaciones. Analizando el lenguaje de los expertos del sector hemos podido constatar que existe una gran cantidad de nuevas voces, términos que se utilizan directamente del inglés y otras que se han adaptado completa o parcialmente. Estas unidades neológicas nacen para etiquetar nuevas formas de hacer turismo, nuevos perfiles de turista, nuevas maneras de promocionar y vender la experiencia turística y de gestionar los establecimientos hoteleros.

Tras la clasificación por áreas temáticas y el análisis de los candidatos a neologismo extraídos de nuestro corpus del lenguaje de los expertos del turismo podemos concluir que el lenguaje de los expertos del turismo está lleno de anglicismos relacionados con la tecnología aplicada al sector. Por otro lado, la mayoría de estos neologismos en el lenguaje profesional del turismo son anglicismos no adaptados en su forma (préstamos puros), si bien en muchos casos estas formas puras coexisten con el préstamo adaptado y son habituales las vacilaciones ortográficas. Asimismo, debido a la alta productividad de algunos lexemas que presentan una alta frecuencia, se están generando nuevas familias de palabras en distintas áreas del sector turístico que presentan un interés investigador. También hemos podido establecer que las unidades neológicas en el lenguaje turístico surgen en cinco áreas temáticas principales: i) formas de hacer turismo o nuevos productos turísticos, ii) nuevos perfiles de turista, iii) marketing, promoción y venta por internet, iv) nuevos modelos de gestión hotelera e v) innovación tecnológica. Esta última categoría temática es la más relevante y la que tiene un mayor impacto actualmente en la gestión turística, si bien el marketing, la promoción y venta también juegan un papel predominante en este sector.

La traducción turística, en tanto que traducción especializada, es un proceso que ha de ser considerado en toda su dificultad. La continua innovación léxica es un hecho incontestable en la industria turística. También tienen un papel destacado otras cuestiones extralingüísticas, como es el hecho de que la lengua inglesa se sitúe como lengua primaria para la formación primaria de términos, lo que repercute directamente en el uso de la neología (la transferencia masiva de anglicismos) y la propia producción neológica de términos en español. Todo ello constituye una dificultad añadida para el traductor de este tipo de textos.

Coincidimos con García Palacios (2015) en que la reflexión fundamental debe girar en torno al uso que realizan los principales actores de las nuevas unidades léxicas. En el caso que nos ocupa, se hallan, por una parte, los especialistas del texto turístico como usuarios directos y generadores de la terminología y por otra, los traductores especializados en el texto turístico, quienes también contribuyen a la difusión de los nuevos términos. Estos últimos deben ser conscientes de la necesidad de ofrecer una coherencia y un equilibrio entre la realidad

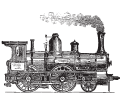

95 
neológica que se deriva del uso especializado y el establecimiento de formaciones aceptables desde el punto de vista lingüístico, pues la traducción de un neologismo en la LO no comporta necesariamente siempre la acuñación de un neologismo en la LM. A la luz de los resultados de nuestro análisis, podemos concluir que el traductor de textos turísticos debe ser consciente de la variada neología léxica perteneciente a diferentes ámbitos del lenguaje del turismo en español, pero en especial, al que hace referencia a la tecnología y la innovación tecnológica. Si bien algunas de estas nuevas voces no son objeto de debate alguno, ya que han prosperado en lengua española y no son exclusivas del lenguaje turístico (web, cluster, selfi, blog...), sí existen unidades léxicas que forman parte de la neología especializada del turismo y que requerirán un esfuerzo adicional por parte del traductor para desentrañar su significado y puede ser necesario que el traductor tenga que recurrir a la creación neológica (gentrificación turística, ryanaización, influenciador de viajes). Sin embargo, debemos puntualizar que es necesario plantearse la pertinencia de la unidad neológica en la traducción (para ello son de gran utilidad los corpus de exclusión y los repertorios de neología, así como los foros de neología) y que ante la opción del anglicismo (voluntourism) existe en ocasiones la posibilidad de poder optar por otras formas ya documentadas (turismo solidario, turismo de voluntariado) que siguen la norma en lo que a formación de palabras en español se refiere.

Este estudio ha pretendido acercar al traductor la realidad neológica del lenguaje turístico de los especialistas, de modo que sirva como una herramienta de ayuda adicional para el traductor de textos turísticos a la hora de la toma de decisiones neológicas durante el proceso de traducción. Una vez observado el comportamiento neológico de los especialistas, la investigación futura podría centrarse en I) analizar en qué medida la formación de los especialistas en el conocimiento de la formación de palabras de las lenguas en contacto puede influir en su opción de uso; II) observar las condiciones que favorecen o dificultan el recurso al neologismo en la traducción de este tipo de textos y iII) la elaboración de propuestas prácticas y concretas de traducción en español de aquellos anglicismos y familias de palabras neológicas más frecuentes en el lenguaje del turismo.

RECIBIDO EN MAYO DE 2017

ACEPTADO EN SEPTIEMBRE DE $20 I 8$ VERSIÓN FINAL DE SEPTIEMBRE DE 2018

\section{REFERENCIAS BIBLIOGRÁFICAS}

Alcaraz Varó, Enrique y Brian Hughes (1999): Diccionario de términos económicos, financieros y comerciales, Barcelona: Ariel.

Alcaraz Varó, Enrique et al. (2006): Diccionario de términos de turismo y ocio, Barcelona: Ariel.

Almela Pérez, Ramón (1999): Procedimientos de formación de palabras en español, Barcelona: Ariel.

Alvar Ezquerra, Manuel (1993): La formación de palabras en español, Madrid: Arco Libros.

Berná Sicilia, Celia (20II): «Phonetic adaptation and derivational morphological development of foreign words in Spanish in the Diccionario Panbispánico de Dudas», en J.L. Cifuentes Honrubia y S. Rodríguez Rosique (eds.), Spanish Word Formation and Lexical Creation, Amsterdam/Philadelphia: John Benjamins, 285-306.

Borrueco Rosa, María (Coord.) (2006): «Sistemas conceptuales en la producción y recepción de textos turísticos. Estudio Aplicado», en La especialización lingüistica en el ámbito del turismo. Sevi1la: Junta de Andalucía. Consejería de Turismo, Comercio y Deporte, 33-51.

Cabré, María Teresa (I992): La Terminologia: al teoria, els mètodes, les aplicacions, Barcelona: Empúries.

- (2006): «La clasificación de neologismos: una tarea compleja», Alfa, 5o/2, 229-250. 
Cabrera Méndez, Marga (2012): «Escribir en internet. Entendiendo el vocabulario del nuevo turismo» en Fundéu B BVA, <http://www.fundeu. es/escribireninternet/entendiendo-el-vocabulario-del-nuevo-turismo/> [Consulta: 23-V-2016].

CAlvi, Maria Vittoria (2006): Lengua y comunicación en el español del turismo, Madrid: Arco Libros.

- (2008): «El lenguaje del turismo: de los textos especializados a la Comunidad del viajero», en Carmen Navarro (ed.), La comunicación especializada, Berlín: Peter Lang, I8I-202.

CARPI, Elena (20I3): «La neología léxica en el discurso del turismo: análisis de blogs profesionales del corpus Linguaturismo 5I», en Luisa Chierichetti y Giovanni Garofalo (eds.) Discurso Profesional y Lingüistica de Corpus, Perspectivas de Investigación, CERLIS Series, 3, 51-7I. <http://dinamico.unibg.it/ cerlis/public/CERLIS_SERIES_3.pdf > [Consulta: 2-VI-2OI6].

Castillo Fadic, M. Natalia (2002): «El préstamo léxico y su adaptación: un problema lingüístico y cultural» Onomazéin, 7, 469-496.

Diccionario de neologismos online. Observatori de Neologia, Universitat Pompeu Fabra, <http:// obneo.iula.upf.edu/spes/> [consulta 23-V-20I6].

Estornell Pons, María (2009): «Neologismos en la prensa: criterios para reconocer y caracterizar las unidades neológicas", Quaderns de Filologia, anejo 70.

- (2013): «Aproximación al léxico del turismo activo: codificación lexicográfica, formación y variación denominativa», Normas. Revista de estudios lingüisticos Hispánicos, 3, 33-55.

García Palacios, Joaquín (2015): «Neología y traducción especializada: ¿un mismo espacio de actuación?», en Alves, I.M; Simões Pereia, E. (ed.) Neologia das Línguas Românicas. São Paulo: Humanitas, 343-366.

Giménez Folqués, David (2015): "Anglicisms in Tourism Language Corpora 2.0.» Procedia Social and Behavioural Sciences, I98, I49-I56.

Gómez Capuz, Juan (2009): «El tratamiento del préstamo lingüístico y el calco en los libros de texto de bachillerato y en las obras divulgativas», Tonos digital. Revista de estudios filológicos, I7. I-24.

GonZÁlEZ, Luis (20I2): «Una propuesta para abordar el conflicto neológico en la traducción» en Cabré Maria Teresa, Bach Carme y Martí Jaume (eds.) Terminología y Derecho: complejidad de la comunicación multilingüe, Institut Universitari de
Lingüística Aplicada, Serie Activitats, 18, 267-280. González-Pastor, Diana María y Miguel Ángel Candel-Mora (20I7): "Creación léxica y anglicismos en el lenguaje de la gestión del turismo en español», E-aesla, 3, 32I-33I.

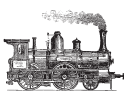

Gotтi, Maurizio (2006): «The language of Tourism as specialized discourse», en Palusci, O. Francesconi, S. (eds.), en Translating Tourism. Linguistic and Cultural representations. Trento: Università di Trento, I5-34.

Guerrero Ramos, Gloria (1995/201o): Neologismos

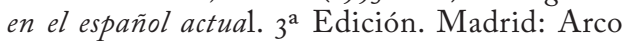
Libros.

GuYot, Jacques (20I0): «La diversidad lingüística en la era de la mundialización», Historia y Comunicación Social, 15, 47-6r.

Hosteltur. Noticias de Turismo. <http://www. hosteltur.com>.

Innvattur. Instituto Valenciano de Tecnologías Turísticas (2OI6): <HTTP://INVATTUR.GVA.ES>

Janssen, Maarten (2009): «Detección de Neologismos: una perspectiva computacional». Debate Terminológico, 5. ISSN I8I3-I867. <http://seer.ufrgs. br/index.php/riterm/article/view/23915/13860>, [consulta: 3-XI- 20I5].

LANG, Mervyn F. (I990): Formación de palabras en español. Morfología derivativa productiva en el léxico moderno, Madrid: Cátedra.

Lorenzo, Emilio (1996): Anglicismos bispánicos, Madrid: Gredos.

Observatori de neologia (----): Diccionario de neologismos online, <http://obneo.iula.upf.edu/ spes $>$. IULA, Universitat Pompeu Fabra.

Sanmartín SÁez, Julia (2009): «El neologismo desde una perspectiva contrastiva: entre lo cognitivo y lo lexicográfico", Revista de Investigación Lingüística, I2, I47-I74.

Segittur. Sociedad Estatal para la Gestión de la InNovación y las Tecnologías Turísticas, $<$ http://www.segittur.es/es/inicio/index.html>.

Termcat (2005): Manlleus $i$ calcs lingüistics en Terminologia, Vic/Barcelona: Eumo Editorial/ TERMCAT.

Tripadvisor (20I6): TripBarometer Global Edition https://www.tripadvisor.com/TripAdvisorInsights/n267o/6-key-travel-trends-2or6, [consulta: I3-XI- 20I6].

Varela Ortega, Soledad (2005): Morfología léxica: la formación de palabras, Madrid: Gredos. 